\title{
Implementing an Urban Public Health Observatory for (Near) Real-Time Surveillance for the COVID-19 Pandemic
}

\author{
Whitney S. BRAKEFIELD ${ }^{\mathrm{a}, \mathrm{f}}$, Nariman AMMAR ${ }^{\mathrm{a}}$, Olufunto OLUSANYA ${ }^{\mathrm{a}}$, \\ Esra OZDENEROL ${ }^{b}$, Fridtjof THOMAS ${ }^{c}$, Altha J. STEWART ${ }^{d}$, Karen C. JOHNSON ${ }^{c}$, \\ Robert L. DAVIS ${ }^{a}$, David L. SCHWARTZ ${ }^{\mathrm{c}, \mathrm{e}, \mathrm{g}}$ and Arash SHABAN-NEJAD ${ }^{\mathrm{a}, 1}$ \\ ${ }^{a}$ University of Tennessee Health Science Center-Oak Ridge National Laboratory \\ (UTHSC-ORNL) Center for Biomedical Informatics, Department of Pediatrics, College \\ of Medicine, Memphis TN, USA \\ ${ }^{b}$ Department of Earth Sciences, University of Memphis, Memphis, TN, USA \\ ${ }^{c}$ Department of Preventive Medicine, UTHSC, Memphis, TN, USA \\ ${ }^{d}$ Department of Psychiatry UTHSC, Memphis TN, USA \\ ${ }^{e}$ Department of Radiation Oncology, UTHSC, Memphis, TN, USA \\ ${ }^{f}$ The Bredesen Center for Data Science, University of Tennessee, Knoxville. TN, USA \\ ${ }^{g}$ Department of Radiation Oncology, University of Texas M.D. Anderson Cancer \\ Center, Houston, TX, USA
}

\begin{abstract}
The COVID-19 pandemic is broadly undercutting global health and economies, while disproportionally impacting socially disadvantaged populations. An impactful pandemic surveillance solution must draw from multi-dimensional integration of social determinants of health $(\mathrm{SDoH})$ to contextually inform traditional epidemiological factors. In this article, we describe an Urban Public Health Observatory (UPHO) model which we have put into action in a mid-sized U.S. metropolitan region to provide near real-time analysis and dashboarding of ongoing COVID-19 conditions. Our goal is to illuminate associations between SDoH factors and downstream pandemic health outcomes to inform specific policy decisions and public health planning.
\end{abstract}

Keywords. Pandemic Surveillance, Data Integration, COVID-19, Urban Health Observatory, Precision Public Health

\section{Introduction}

The COVID-19 pandemic is an international health crisis; it represents a leading direct and indirect cause of death in many countries. This burden falls disproportionally onto populations facing social disadvantage. Our prior work has explored associations between social determinants of health $(\mathrm{SDoH})$ and several health outcomes (e.g., asthma, diabetes, and hospital readmission) [1-4]. Integrating SDoH indicators with the relevant health indicators is now an integral step for the implementation of intelligent public health surveillance solutions [5]. Health Intelligence [6] can assist researchers to explore the causal pathways between drivers (e.g., SDoH) and outcomes (e.g., COVID-19

\footnotetext{
${ }^{1}$ Corresponding Author, Arash Shaban-Nejad, Centre for Biomedical Informatics, 492R-50 N. Dunlap Street, Memphis, TN 38103; E-mail: ashabann@uthsc.edu.
} 
positive cases, COVID-19 morbidity, and mortality) as well as correlations between the different outcomes. The process involves classifying the collected data into drivers and outcomes and studying to what extent we can identify or develop interventions to mitigate drivers that lead to the undesired outcomes. In this paper, we describe an Urban Public Health Observatory (UPHO) (Figure 1) for (near) real-time surveillance of the current pandemic. The UPHO assists public health authorities, epidemiologists, and researchers to collect data from several resources, foster the integration of surveillance data consistently across jurisdictions to estimate the incidence and prevalence of different health conditions, as well as related risk factors.

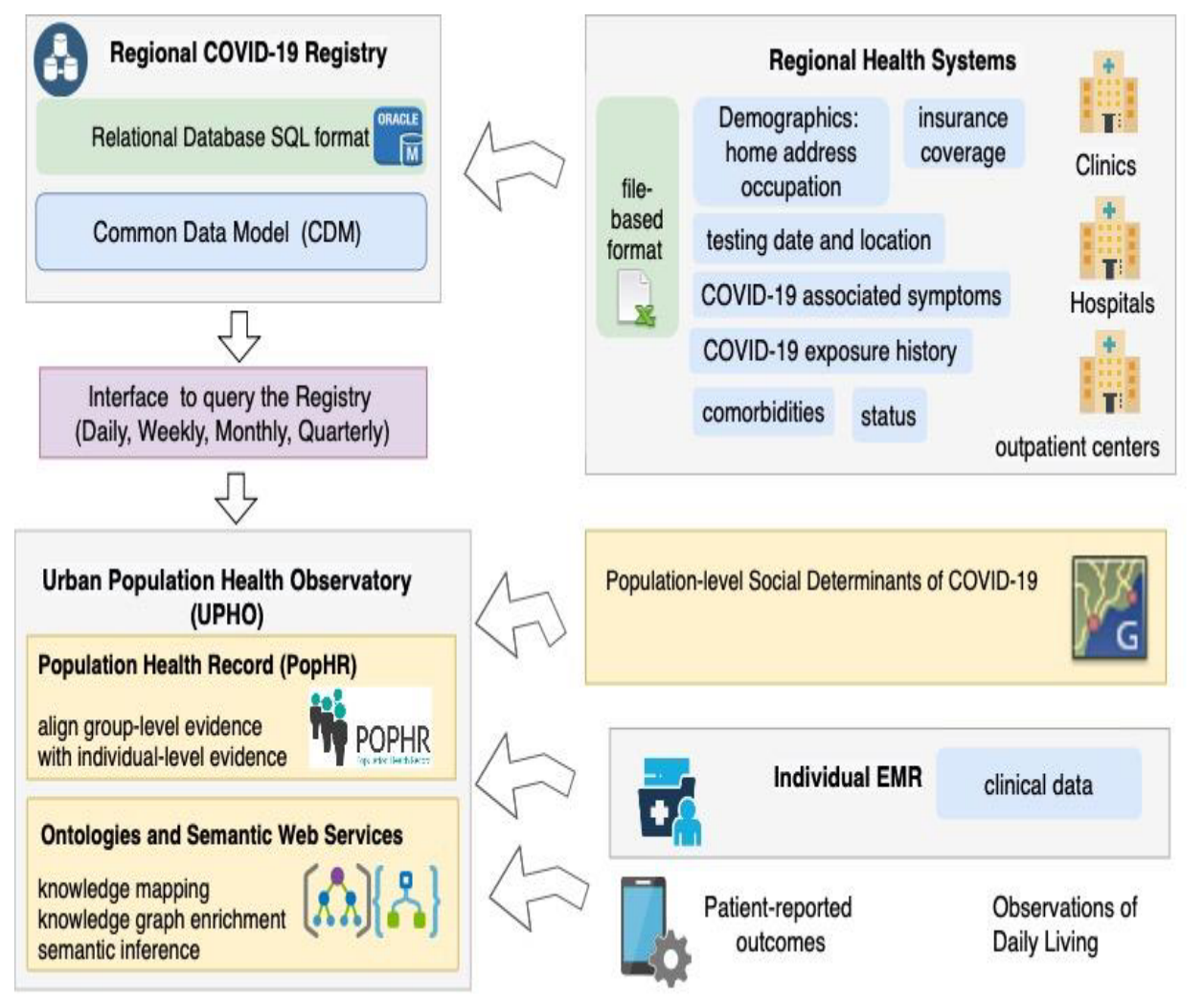

Figure 1: An abstract representation of the Memphis Urban Public Health Observatory that integrates data from several sources, including individual-level COVID-19 indicators collected through a regional registry, population-level SDoH indicators, clinical data in the patients' EMRs, and patient-reported outcomes.

As demonstrated in Figure 1, UPHO integrates data from several sources, including individual-level COVID-19 indicators collected through a regional registry, populationlevel SDoH indicators, clinical data in patients' EMRs, and patient-reported outcomes. The COVID-19 registry systematically collects Individual-level COVID-19 indicator variables. UPHO aligns those individual-level indicators with population-Level Social Determinants of COVID-19 and data collected by tracking observations of daily living. In this article, we provide a classification of Social Determinants of COVID-19, and explain how they have been collected, and integrated to be used for intelligent queryanswering to formally interrogate hypothesis-driven research questions. 


\section{Methods and Results}

\subsection{Study Area, Population and Study measures}

A Metropolitan Statistical Area (MSA) consists of Core Based Statistical Areas that have a core urbanized area with at least 50,000 people. It is comprised of the central county that contains the core urban area and all adjacent counties that are linked to that county through social or economic ties, typically measured by commuting patterns. The registry currently collects data from several regional health systems in the Memphis MSA, which includes counties in Tennessee, Mississippi, and Arkansas. Memphis MSA has a land area of about 4,985 square miles [7]. According to the U.S. Census Bureau, Memphis MSA has a population of $1,350,064$ and the different counties are affected to quite different extend, see Fig. 2. 43\% of the population is white, $47 \%$ is African American, 6\% is Hispanic or Latino, $2 \%$ is Asian, and $2 \%$ is two or more races. $52 \%$ of the Memphis MSA population are females and $48 \%$ are males. For this study, SDoH and social distancing metrics represent the predictor variables while COVID-19 indicators serve as the outcome variables. As for the outcome variables, individual-level indicators include the date of testing, testing locations, residential address, medical insurance coverage, COVID-19 symptoms and exposure history, comorbidities, and occupation as a health worker or first responder. We discuss predictor variables in the following section.

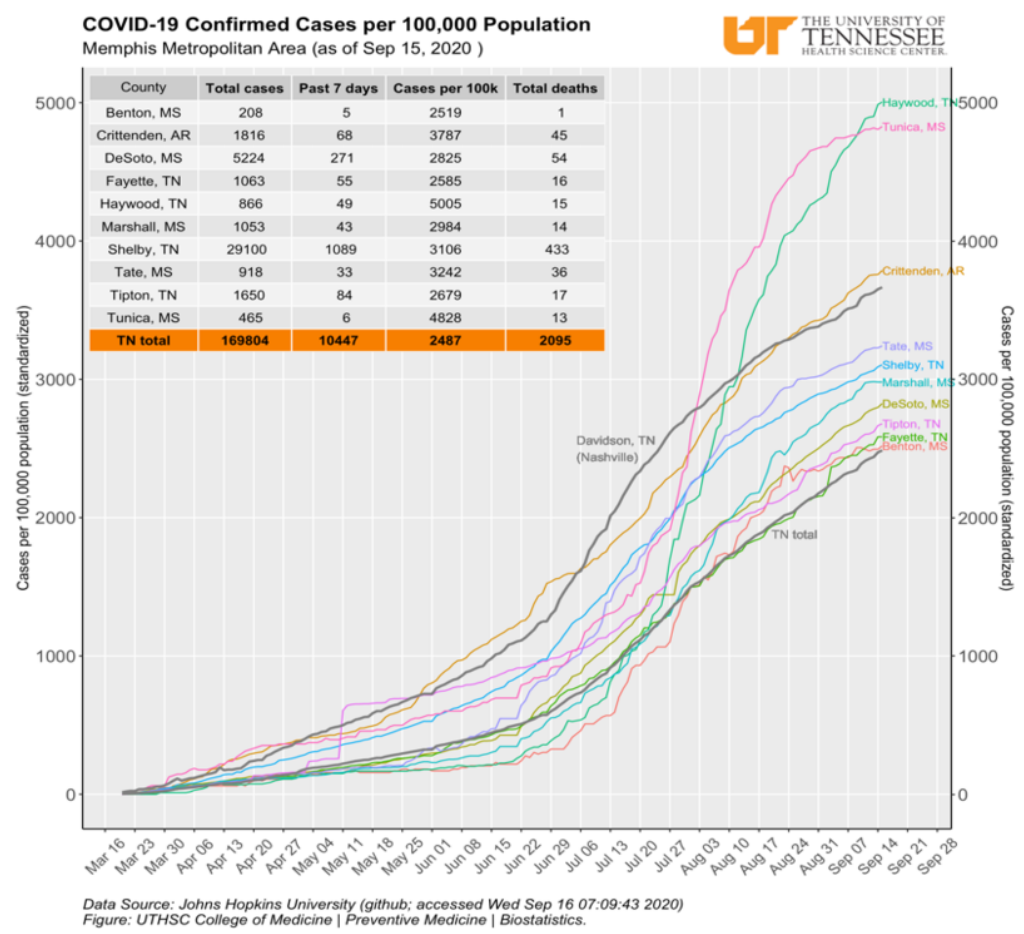

Figure 2: Counties in the Memphis Metropolitan Area and their COVID-19 confirmed cases per 100,000 population as the pandemic unfolds.

\subsection{Social Determinants of COVID-19}

The Center for Disease Control and Prevention (CDC) provides a taxonomy for SDoH that is comprised of 6 domains: economic stability (e.g., income), education (e.g., 
educational attainment and graduation rates), health and healthcare access ( access to health and how well an individual or group understands the health information to make the appropriate decisions), social and community context (variables reflect the social setting that an individual resides and their community involvement), demographics (e.g., race/ethnicity, sex, age), and neighborhood and built environment (variables that relate to the physical surrounding environment and have the potential to overlap other domains) $[8,9]$. SDoH are associated with COVID-19 transmission and mortality [10-17]. SDoH overlap and interact in real-world setttings, requiring careful disentanglement of their individual downstream inpact on health outcomes such as COVID-19 spread. This project directly addresses this complexity, providing a crucial first step towards intelligent surveillance solutions to assist pandemic recovery [5]. We classify the SDoH associated with the COVID-19 pandemic into their 6 domains [8, 9]: i) SDoH that affect access to resources, $i$ i) SDoH that increase disease exposure, susceptibility, and severity; iii) SDoH affecting adherence to policies; iv) SDoH that are community characteristics; v) SDoH that enable increasing awareness, knowledge dissemination, and health education; and vi) $\mathrm{SDoH}$ specific to neighborhood and built environment which can impact COVID-19 associated comorbidities

\subsection{Data Collection, Analytics, and Visualization}

As shown in Fig. 1, UPHO collects 3 types of data, SDoH, social distancing metrics, and COVID-19 related outcomes, and aggregates them at Census Block Group (CBG) level. To obtain SDoH variables, we utilize the U.S. Census Bureau 2018 American Community Survey data [18]. We collect social distancing metrics since social distancing and shelter-in-place were among the most effective early interventions during the pandemic. For that purpose, we utilize the publicly available SafeGraph [19] movement behavior dataset, taking into account the phased interventions announced in the host MSA area starting from March 30, 2020, through a phased opening, including how often people visit specifically categorized public locations, the duration of their stay, where they come from, etc. This CBG-level data is collected anonymously from personal mobile phone use. We utilize the dataset to assess relationships among population movement behavior, transportation patterns, and COVID-19 transmission rates.

UPHO enables conducting exploratory geospatial analyses of COVID-19 transmission patterns, including neighborhood-level clusters and hot spots. We then apply machine learning to test novel epidemiological models by linking COVID-19 outcomes with the $\mathrm{SDoH}$ and social distancing metrics at the $\mathrm{CBG}$ level for the identification of the geographic, sociodemographic, and disease-specific risk factors predictive of COVID-19 positivity, the spread of COVID-19 across the MSA region, and downstream clinical outcomes.

UPHO integrates multidimensional SDoH and epidemiology data and makes them accessible via a public dashboard. Future directions will focus on further implementation of the dashboard that queries the observatory through its API and visualizes the results at different geographical resolutions. The dashboard can be used to answer several research questions such as i) to what degree is the disease spread associated with specific actionable or measurable determinants, ii) to what degree public adherence to pandemic mitigation policies is influenced by these determinants, and iii) how can data-driven insights directly inform policy changes to accommodate different populations and areas, especially as most cities prepare to enter re-opening phases. 


\section{Conclusion}

Our UPHO provides first-of-kind insights for immediate and long-term health policy response to COVID-19. The application of the dashboard is not limited for use to only scientific investigators, epidemiologists and healthcare professionals. Measures of SDoH from the dashboard could also be accessible to the general public in the form of neighborhood-level data as well as government officials to inform policymaking. In addition to the epidemiological surveillance of infectious diseases such as COVID-19, the UPHO may also have utility for monitoring and learning about chronic diseases e.g. cancers in the urban population. Overall, these outcomes reduce health disparities, achieve health equity, and improves urban population health. The platform provides a reproducible, durable, and scalable model for data-driven, socially-informed policymaking for recovery and future-readiness for large-scale pandemic events.

\section{References}

[1] Shin EK, Mahajan R, Akbilgic O, Shaban-Nejad A. Sociomarkers and biomarkers: Predictive modeling in identifying pediatric asthma patients at risk of hospital revisits. NPJ digital medicine. 2018;1(1):1-5.

[2] Shin EK, LeWinn K, Bush N, Tylavsky FA, Davis RL, Shaban-Nejad A. Association of maternal social relationships with cognitive development in early childhood. JAMA network open. 2019;2(1):e186963.

[3] Shin EK, Shaban-Nejad A. Urban decay and pediatric asthma prevalence in memphis, tennessee: Urban data integration for efficient population health surveillance. IEEE Access. 2018;6:46281-46289.

[4] Shin EK, Kwon Y, Shaban-Nejad A. Geo-clustered chronic affinity: Pathways from socio-economic disadvantages to health disparities. JAMIA open. 2019;2(3):317-322.

[5] Shaban-Nejad A, Lavigne M, Okhmatovskaia A, Buckeridge DL. PopHR: a knowledge-based platform to support integration, analysis, and visualization of population health data. Ann N Y Acad Sci. 2017 Jan;1387(1):44-53.

[6] Shaban-Nejad, A., Michalowski, M. \& Buckeridge, D.L. Health intelligence: how artificial intelligence transforms population and personalized health. npj Digital Med . 2018;53(1):1-2.

[7] Census profile: Memphis, TN-MS-AR Metro Area [Internet]. Census Reporter. 2020 [cited 2020 Sep 1]. Available from: https://censusreporter.org/profiles/31000US32820-memphis-tn-ms-ar-metro-area

[8] Social Determinants of Health | Healthy People 2020 [Internet]. Healthypeople.gov. 2020 [cited 2020 Sep 6]. https:/www.healthypeople.gov/2020/topics-objectives/topic/social-determinants-of-health

[9] About Social Determinants of Health (SDOH) [Internet]. Centers for Disease Control and Prevention. 2020 [cited 2020 Sep 6]. https://www.cdc.gov/socialdeterminants/about.html.

[10] Fielding-Miller RK, Sundaram ME, Brouwer K. Social determinants of COVID-19 mortality at the county level. medRxiv [Preprint]. 2020 Jul 1[cited 2020 July 1]. Available from: https://www.medrxiv.org/content/10.1101/2020.05.03.20089698v2 doi: 10.1101/2020.05.03.20089698.

[11] Cyrus E, Clarke R, Hadley D, Bursac Z, et al. The impact of COVID-19 on African American communities in the United States. medRxiv [Preprint]. 2020 May 19 [cited 2020 July 1]. Available from: https://www.medrxiv.org/content/10.1101/2020.05.15.20096552v1 doi: 10.1101/2020.05.15.20096552.

[12] Bai S, Jiao J, Chen Y. Mapping the Intercounty Transmission Risk of COVID-19 in New York State. SSRN [Prepint]. 2020 [cited 2020 July 1]. Available from: https://ssrn.com/abstract=3582774

[13] Chen Y, Jiao J, Bai S, Lindquist J. Modeling the spatial factors of COVID-19 in new york city. SSRN [Prepint]. 2020 [cited 2020 July 1]. Available from: https://ssrn.com/abstract=3606719

[14] Millett GA, Jones AT, Benkeser D et al. Assessing differential impacts of COVID-19 on black communities. Ann Epidemiol. 2020 Jul;47:37-44.

[15] Jiwani SS, Antiporta DA. Inequalities in access to water and soap matter for the COVID-19 response in sub-Saharan Africa. International Journal for Equity in Health. 2020 Dec;19:1-3.

[16] Qiu Y, Chen X, Shi W. Impacts of social and economic factors on the transmission of coronavirus disease 2019 (COVID-19) in China. Journal of Population Economics. 2020 Apr 6:1.

[17] Del Brutto OH, Mera RM, Recalde BY, Costa AF. Social Determinants of Health and Risk of SARSCoV-2 Infection in Community-Dwelling Older Adults Living in a Rural Latin American Setting. Journal of Community Health. 2020 Jul 15:1-6.

[18] About the Survey [Internet]. The United States Census Bureau. 2020 [cited 2020 Sep 10]. Available from: https://www.census.gov/programs-surveys/acs/about.html

[19] SafeGraph. [Internet]. 2020 [cited 2020 Sep 10]. Available from: https://docs.SafeGraph.com/docs/faqs 